\title{
Secure Multiparty Quantum Computation with (Only) a Strict Honest Majority
}

\author{
Michael Ben-Or \\ The Hebrew University \\ benor@cs.huji.ac.il
}

\author{
Claude Crépeau \\ McGill University \\ crepeau@cs.mcgill.ca
}

\author{
Daniel Gottesman \\ Perimeter Institute for Theoretical Physics \\ dgottesman@ @erimeterinstitute.ca
}

\author{
Adam Smith \\ Weizmann Institute of Science \\ adam.smith@weizmann.ac.il
}

October 23, 2018

\begin{abstract}
Secret sharing and multiparty computation (also called "secure function evaluation") are fundamental primitives in modern cryptography, allowing a group of mutually distrustful players to perform correct, distributed computations under the sole assumption that some number of them will follow the protocol honestly. This paper investigates how much trust is necessary - that is, how many players must remain honest - in order for distributed quantum computations to be possible.

We present a verifiable quantum secret sharing (VQSS) protocol, and a general secure multiparty quantum computation (MPQC) protocol, which can tolerate any $\left\lfloor\frac{n-1}{2}\right\rfloor$ cheaters among $n$ players. Previous protocols for these tasks tolerated $\left\lfloor\frac{n-1}{4}\right\rfloor$ and $\left\lfloor\frac{n-1}{6}\right\rfloor$ cheaters, respectively. The threshold we achieve is tight — even in the classical case, "fair" multiparty computation is not possible if any set of $n / 2$ players can cheat.

Our protocols rely on approximate quantum errorcorrecting codes, which can tolerate a larger fraction of errors than traditional, exact codes. We introduce new families of authentication schemes and approximate codes tailored to the needs of our protocols, as well as new state purification techniques along the lines of those used in fault-tolerant quantum circuits.
\end{abstract}

\section{Introduction}

Secure multiparty computation has been studied extensively in the classical setting (see [17] for a survey) and was extended to the quantum setting by [12]. A secure quantum multiparty protocol (or secure function evaluation) allows $n$ participants $P_{1}, \ldots, P_{n}$ to compute an $n$ input quantum circuit where each player $P_{i}$ is responsible for providing one of the input states. The output of the circuit is broken into $n$ components $H_{1} \otimes \ldots \otimes H_{n}$ and $P_{i}$ receives the output
$H_{i}$. Note that the inputs are arbitrary (possibly entangled) quantum states and each player simply has his input in his possession - he does not need to know its classical description. Informally we wish to achieve the same functionality as if each player were to hand his input to a trusted third party who would evaluate the circuit and distribute the outputs. Moreover we wish to do so even when up to $t$ players are faulty.

In the quantum setting it seemed at first that the best one could hope for is to tolerate $t<n / 4$ faulty players simply because (exact) quantum error correcting codes (QECC) cannot recover from more errors. Indeed the best previously known verifiable quantum secret sharing protocol can tolerate $t<n / 4$ faulty players, and the best secure quantum multiparty protocol tolerates only $t<n / 6$ faults $[12]$. However, approximate QECCs exist [13] that can recover (with high probability) from the corruption of $t<n / 2$ shares, and their discovery paved the way to this paper.

Main Result Assuming pairwise quantum channels and a classical broadcast channel between $n$ players, there exists a universally composable, statistically secure multiparty quantum computation protocol, that tolerates an adaptive adversary controlling up to $t<n / 2$ faulty players. The complexity of the protocol is polynomial in the number of players and the size of the circuit.

Note: Tolerating $t \geq n / 2$ faulty players is not possible in our model without computational assumptions. This follows, for example, from the impossibility of unconditionally secure quantum bit commitment for two players (see [15] for a recent discussion).

In our setting, universally composable classical secure multiparty computation is possible [23, 7, 20] and, crucially, the proofs of composability hold even in the quantum UC

\footnotetext{
${ }^{1}$ The preliminary version of [12] claims that the $n / 4$ bound is tight for VQSS; however, the bound holds only for errorless protocols. See [13] for a discussion.
} 
model [6]. One strategy we use extensively is to reduce the quantum multiparty computation to a secure computation on classical keys.

Protocol Overview Our protocol follows the basic "shareand-compute" paradigm of classical distributed protocols. Players use a quantum version of verifiable secret sharing (called VQSS) to distribute an encoding of their input. They then perform the circuit, gate by gate, on these encoded values. The circuit's outputs are then sent to appropriate players by opening the VQSS.

The structure of the verifiable sharing is similar to classical protocols, with several important differences. First, the encoding used in the VQSS combines error-correction and authentication in a novel way. Authenticating quantum data requires encryption, and new tools are required to manipulate encrypted data. In particular, we can often push the complexity of the quantum computation into a classical computation on the authentication (or encryption) keys this computation can be performed with classical tools. One of our contributions is a family of self-dual authentication codes, which make these classical computations simpler to represent.

Second, the error-correcting code underlying the protocols is an "approximate" code which tolerates any $t<n / 2$ errors, similar to that in [13]. In order to perform a full (dense) set of operations fault-tolerantly on these encodings, we develop a procedure for purifying encodings of "Toffoli states", whose creation is sufficient to perform a Toffoli gate.

Finally, because quantum data cannot be cloned, the dealer does not use his actually data in the sharing protocol until after the sharing is successfully completed - he then inputs his data via teleportation.

This Abstract The basic authentication code, and operations required on authenticated data, are described in Section 2 Sections 3 , 4 and [5 build up the pieces necessary for VQSS. Section 3 extends the authentication scheme to verified state authentication, in which a dealer can prove to another player that a message is correctly authenticated via a key shared among all players. The next step is weak VQSS, which plays the role of a classical commitment scheme: the dealer shares a state in such a way that he cannot change it, but may refuse to open it at a later stage (Section 4). Section 5 explains the final layer needed for VQSS. Finally, Sections 6 and 7 give the purification procedure for Toffoli states and a sketch of the simulation argument showing that the whole protocol is secure.

The Network Model We assume a synchronous network (with rushing) in which pairwise secure quantum channels exist between any two players, and a classical broadcast channel connects all players.
The Adversary We assume that the adversary is computationally unbounded, and that she fully coordinates the actions of all faulty players. She may corrupt players adaptively during the course of the protocol. There are two limitations to this: (1) At most $t<\frac{n}{2}$ players may become faulty during the course of the computation; (2) the adversary has access only to the information of the corrupted players she currently controls. We call the non-faulty players honest.

All protocols we present have a success probability exponentially close to one (also called an exponentially good probability) in some security parameter 2 .

\section{Quantum Authentication}

In our construction of Multiparty Quantum Computation (MPQC) we use a quantum authentication scheme (QAS), such as that proposed in [3]. Any QAS based on a quantum CSS code can be used, but some later protocols become simpler when the QAS is based on a self-dual code. Therefore our first contribution is a family of self-dual Quantum Authentication Schemes. We will build a scheme which is exponentially secure in some arbitrary security parameter $m=2 d+1$, where $d$ is a parameter of the code. The scheme is not optimal in terms of redundancy or error probability, but it is sufficient for our purposes.

When using this scheme in the computation, we will assume that $m$ is larger than the security parameter of the Multiparty Computation times the number of players. Let $p$ be a prime, $m<p<2 m$. All the algorithms we propose will manipulate qudits in $\mathbb{Z}_{p}$.

The scheme will be based on a classical key which will be composed of two parts: $k_{1}, \ldots, k_{m} \in_{R}\{ \pm 1\}$ and a string $x \in R\{0,1\}^{2 m \log _{2}(p)}$. The dealer will then apply two transformations:

First, in a way quite similar to the stretched polynomial code, the dealer will apply

$$
\left|S_{a}\right\rangle \rightarrow p^{-d / 2} \sum_{\substack{d e g(f) \leq d \\ f(0)=a}}\left|k_{1} \times f\left(\alpha_{1}\right), \ldots, k_{m} \times f\left(\alpha_{m}\right)\right\rangle,
$$

where $\alpha_{1}, \ldots, \alpha_{m} \in \mathbb{Z}_{p}$ are distinct nonzero points known to all players.

Then, very much like the Quantum Authentication Code of [3], the dealer will encrypt the state by applying a random Pauli operation on each part of the state. This will create a stretched and shifted polynomial-like code. The encryption will be denoted as $E_{x}$, so we will have $\psi=E_{x} A_{k}(\phi)$.

\footnotetext{
${ }^{2}$ As proved in [12], Verifiable Quantum Secret Sharing and Multiparty Quantum Computation with more than $n / 4$ faulty players must have some probability of failure. Therefore, the Multiparty Computation protocol we present will have an exponentially small probability of failure in some security parameter. Let $S$ be the value of this parameter, and $C$ the size of the quantum circuit we want to securely evaluate. Our algorithms will be polynomial $n, S$ and $C$. Setting a security parameter $s=S+n^{2}+\log C+O(1)$ in all our subprotocols is sufficient to guarantee that the overall failure probability will be bounded by $2^{-S}$.
} 
Note that the authentication (without the encryption) is self-dual. To see this, we apply the Fourier transform on the code transversely as in [1], getting Fourier $\left(\left|S_{a}\right\rangle\right)=$ $p^{-1 / 2} \sum_{b} \omega^{a b}\left|S_{b}\right\rangle$. This gives:

$$
\left|S_{b}^{\prime}\right\rangle=p^{-d / 2} \sum_{\substack{d e g(f) \leq d \\ f(0)=b}}\left|k_{1}^{-1} \times f\left(\alpha_{1}\right), \ldots, k_{m}^{-1} \times f\left(\alpha_{m}\right)\right\rangle
$$

which is equal to $\left|S_{b}\right\rangle$, as $k_{i}^{-1}=k_{i}$. The combined code $E_{x} A_{k}$ remains self dual - the Fourier transform on $E_{x}$ is equivalent to a change in the classical key $x$.

Security of the Quantum Authentication Scheme After the encoding, an adversary can try to tamper with the information as she likes, but without knowledge of $(x, k)=K$.

Finally the receiver takes as input the system $\rho^{\prime}$, and tries to return to the encoded state based on $k, x$. We will apply definitions 1 and 2 of [3], and say that the receiver's output lies in a Hilbert space $M \otimes V$, where $\mathrm{M}$ has a size of $m$ qubits (the size of the original state) and $V$ is a Hilbert space of dimension 2, with basis states $|\mathrm{ac}\rangle,|\mathrm{re}\rangle$. Define projectors

$$
\begin{aligned}
P_{1}^{|\psi\rangle}= & |\psi\rangle\left\langle\psi\left|\otimes I_{V}+I_{M} \otimes\right| \text { re }\right\rangle\langle\text { re }| \\
& -|\psi\rangle\langle\psi|\otimes| \text { re }\rangle\langle\text { re }| \\
P_{0}^{|\psi\rangle}= & \left(I_{M}-|\psi\rangle\langle\psi|\right) \otimes|\mathrm{ac}\rangle\langle\mathrm{ac}|
\end{aligned}
$$

Lemma 2.1. In expectation over $k$ and $x$, for any encoded state and for any action taken by the adversary, the receiver's output has exponentially good fidelity to the space spanned by $P_{1}^{|\psi\rangle}$ 3. If the adversary did not change the authenticated state the output will be the original state tensored with $|\mathrm{ac}\rangle$.

The proof of the lemma appears in the final version.

Actually, this security definition is not quite sufficient for our purposes, since we need the authentication to remain secure in a variety of contexts. We adopt the Universally Composable definition of [18] with a TTP: That is, the sender passes the state to the TTP. The adversary then gets to decide whether the TTP gives the correct state to the receiver, or instead the state $|r e\rangle$. Hayden et al. [18] show that the class of QAS described in [3], including the code above, remain secure with respect to this stronger definition.

We also need the authentication scheme to remain secure when it is applied to many states authenticated with the same $k$ but different $x$ 's. The proof is essentially the same: we can treat the combined system as a single large authentication scheme which fails if even one of the states fails the authentication test. It is again sufficient to consider attacks where the adversary applies a Pauli matrix, and the argument above shows that she is likely to be caught if she attacks even a single state in this way.

\footnotetext{
${ }^{3}$ In particular, with exponentially good probability over $k, x$, the fidelity is exponentially close to 1 .
}

Operations on Authenticated Quantum Data A key advantage of the code we present is that it is possible to perform Clifford operations on coded data when one party holds the classical keys and the other party holds the data. In all cases, we can do this by performing transversal operations on the quantum state (that is, separate quantum gates on each share), and some corresponding transformation of the encryption key $x$. For instance, the Fourier transform can be done applying the Fourier transform transversally and changing the key $x=\left(x_{0}, x_{1}\right)$ to $x^{\prime}=\left(x_{1}, x_{0}\right)$, and the $\bmod p$ SUM gate by transversal SUM gates while transforming the keys $x=\left(x_{0}, x_{1}\right), y=\left(y_{0}, y_{1}\right)$ to $x^{\prime}=$ $\left(x_{0}, x_{1}-y_{1}\right), y^{\prime}=\left(x_{0}+y_{0}, y_{1}\right)$. Measurement can be performed by measuring each qudit to get a classical word, which can be decoded with the help of the key.

A few properties of the code are:

1. The new states are still correctly authenticated.

2. Only the correct gate leaves the states correctly authenticated (since a different gate would require a different transformation of $x$ ).

3. Performing any of these operations does not give any new data on the keys. This is important in the case of CNOT, where knowing the key $x^{\prime}$ of one of the states after the operation does not give any information on the key $y^{\prime}$ of the other state.

Measuring an authenticated state according to the standard basis yields a random codeword which is multiplied and shifted. Changes to the codeword without knowledge of the keys is equivalent to applying $X$ operations on the quantum state, and has the same probability of getting caught.

Handling keys In the following sections we will want to manipulate the classical keys in many ways. We will use an imaginary classical Trusted Third Party, which implements classical multiparty computation. From now on, all classical keys of authenticated data will be sent immediately to this Third Party, which will tell the players the meaning of their actions based on those keys. While such a TTP does not exist, we can simulate it using (for example) the classical multiparty computation of [23].

\section{Verified Quantum State Authenti- cation}

In this section, we force the dealer to send each honest player a correctly authenticated message, using the QAS of section 2. A dealer who does not comply will be revealed as faulty in front of all the players and is kicked out. In the first subsection, we show how to force the dealer to send correctly authenticated zero states to every honest player. We later 
transform the zero states to EPR pairs shared between players, and then pass other states using quantum teleportation. The algorithms succeed only with high probability.

Sending "Verifiable" Authenticated Zeros Let $D$ be a dealer, who should send states of the form $A u t h_{(k, x)}(|0\rangle)$ to all players. The problem is that later in the protocol, players are required to present states which have been authenticated by the dealer. There would then be no way to distinguish between an honest player who was originally given bad states by a faulty dealer and a corrupted player who changed the states she received from an honest dealer. To solve this problem we incorporate the following protocol, which guarantees that with exponentially good probability either the dealer is caught, or every honest player has a large number of $|0\rangle$ states authenticated by the dealer:

Protocol Zero-Share (Dealer $D, r$-copies to each player)

1. $D$ chooses one random key $k$, and creates many states of the form Auth $_{(k, x)}(|0\rangle)$ for many different $x$ 's (all $x$ 's are chosen at random).

2. $D$ sends each player many $((r+2 s)(t+1))$ such states.

3. $D$ sends all the keys to the classical TTP.

4. Each player $P_{i}$ performs purification on his states. A purity testing protocol for zeros which spends $2 s$ states is given later. The results of the measurements are sent to the $T T P$.

5. The $T T P$ returns each player a bit indicating whether everything was alright with her states. This gives fidelity 4 of $1-p^{-s}$ to the statement that either $P_{i}$ 's states are authenticated zeros or the dealer is caught by $P$.

6. For each player $i$, if $P_{i}$ caught the dealer she complains about him. If there are more than $t$ complaints the dealer is faulty.

7. Each player who did not complain distributes $r+2 s$ of her states to each player who did complain.

8. Each complainer $P_{i}$ does the following: for all $j$, using the zero purity test protocol and with the help of the TTP (as before $P_{i}$ only measures and the TTP tells him if the states are good), go over the states you got from $P_{j}$. Find a player $P_{j}$ who you can trust (i.e., $P_{j}$ gave $P_{i}$ states which were correctly authenticated by the dealer).

\footnotetext{
${ }^{4}$ In calculating the fidelity we assume that all the authentication checks succeeded.

${ }^{5} \mathrm{~A}$ more formal definition can be cast by letting $P_{i}$ output $|\mathrm{ac}\rangle, \mid$ re $\rangle$ as before and then we have high fidelity to the state of authenticated zeros tensored with $|\mathrm{ac}\rangle$ or anything else tensored with $|\mathrm{re}\rangle$
}

Lemma 3.1. In the last stage of the protocol, with high probability every honest player will have zero states which were authenticated by the dealer.

Proof. At most $t$ players complained about the dealer in step 6. This means that at least one honest player got authenticated zeros, and she will pass them to all the complainers. Therefore, if after the last step a player complains that she doesn't have any authenticated states she is faulty.

Lemma 3.2. The adversary has no new information about the key $k$ used by the dealer or about the $x$ 's in the surviving zero states.

Proof. For all $i$, all the measurements made by $P_{i}$ give random values, and players are only told by the TTP that the check succeeded.

Note that the protocol has an exponentially low probability to fail completely. This could happen (for example) if the dealer sends non-zero states, but a single honest player is fooled by the dealer. This will mean that the dealer will not be considered faulty in step 6 , and all the honest players will fail in the last step.

The protocol requires a method of testing that a set of states are (close to) correctly authenticated zeros. We present such a zero purity test in appendix A

Generating Authenticated EPR pairs To share an authenticated EPR pair with the dealer, $P_{i}$ takes two authenticated zeros, and using the classic $T T P$, performs a transversal Fourier on one of them, and then a SUM. $P_{i}$ then sends $D$ one half of the pair.

In order to see the security of the protocol we need to look at two cases:

1. $P_{i}$ is honest but the dealer is faulty: $P_{i}$ holds zeros which were authenticated by the dealer (as the dealer was not kicked out of the protocol Zero-Share). The rest of the protocol depends on $P_{i}$.

2. The dealer is honest but $P_{i}$ is faulty: The EPR pairs which are authenticated by the dealer will be used to pass information from the dealer to $P_{i}$ using quantum teleportation. A faulty $P_{i}$ could send the dealer a state which is not part of an EPR pair (say by destroying the other half or passing some junk), but this does not add cheating power, as it is equivalent to destroying the data the dealer is trying to give to $P_{2}^{6}$.

\footnotetext{
${ }^{6}$ As we saw, the dealer can not fail this protocol. Therefore, after using it, $P_{i}$ will be considered responsible if anything goes wrong. This alone makes the protocol secure against a faulty $P_{i}$ and an honest dealer.
} 


\section{Weak Quantum Secret Sharing (WQSS)}

Weak Secret Sharing [22, 23] is a protocol with two phases. It provides similar functionality to a classical string commitment scheme, replacing computational assumptions with the cooperation of the honest majority. In the first phase, the dealer shares a (quantum) state (the secret), among all the players, such that the faulty players have no information about the state. In the second phase, the (quantum) data is sent to a reconstructor (sometimes called the receiver), who reconstructs the secret. We demand that if the dealer is honest the reconstructor can reconstruct the secret shared. If the dealer is faulty during the sharing phase some state must still be determined. However, if the dealer is faulty during the reconstruction phase the bad players can make sure that no state is reconstructed. In the case where no state is reconstructed the reconstructor will know that the dealer is faulty. At the end of the sharing phase, the players have a state encoded in the quantum error-tolerant secret sharing scheme of [13], but with an additional security guarantee in the case where the dealer is faulty. As before, we only want our protocol to succeed with high probability.

We give a formal definition using a TTP:

1. The dealer $D$ sends $T T P$ a state $\rho$, or no state at all. If D did not send a state the TTP notifies all the players that this is the case and the protocol ends.

2. Otherwise, at the reconstruction phase, a reconstructor $R$ is chosen.

3. If $D$ is honest, the $T T P$ sends the state $\rho$ to $R$. If $D$ is faulty, she can tell the TTP not to send the state. In this case the TTP tells the reconstructor that $D$ is faulty.

The difference between this variant and Verifiable Secret Sharing lies in step 3 of the definition, where $D$ has a chance to ruin a previously shared state.

Protocol Before the protocol starts (this will be a prerequisite to all our protocols from now on) we assume that the dealer has some secret authentication key $k_{\text {dealer }}$. The dealer will use this authentication key with many random encryption keys.

We maintain the invariant that an honest player will never (with high probability) think that another honest player is faulty. Therefore if more than $t$ players blame the dealer, she is truly faulty and can be kicked out of the protocol.

We give the detailed sharing protocol for WQSS in appendix $B$ The outline of the procedure is as follows:

1. The dealer encodes a number of zero states using a quantum polynomial code, and transmits the shares to the players using the authenticated channel from section 3
2. The players and the classical TTP collectively test that the states are zeros, and are correctly encoded via transversal random sums.

3. The players use the shared zero states to create a shared EPR pair. Half of it is returned to the dealer, who decodes it and teleports his state through the EPR pair.

The protocols we present later use the WQSS but will never use its reconstruction. Therefore we present a naive protocol which relies on revealing the keys. Reconstructing the secret can be made by sending all the quantum data to the reconstructor, as well as the key $k$ and the relevant computed $x$ keys. The reconstructor will open up the authentication of all the states, measure the second qubit ( $|\mathrm{ac}\rangle$ or $|\mathrm{re}\rangle)$ and use only the correctly authenticated points. If they are all from a degree $t$ polynomial code she will reconstruct $|\psi\rangle$; otherwise the dealer is faulty. This sort of reconstruction, however, spoils the secrecy of key $k_{\text {dealer }}$, which we need throughout the entire quantum computation. Therefore we will not use the WQSS as presented here. Still, for completeness of the paper we temporarily assume this naive reconstruction, and discuss the security of the protocol. We also assume the reconstructor is honest, because she gets all the data anyway.

Lemma 4.1. If the dealer is honest, the protocol is secure.

Proof. We first prove that the protocol works, and then prove the secrecy of the data. As the dealer is honest, encoded zeros are being sent by the established authenticated channels. With high probability, all the measurement results which are sent to the TTP are either the right results or they will be discarded (because of the authenticated channels). This means that with high probability an honest dealer will pass the test done by the classical TTP.

In the final step, the dealer will get a state encoding half an EPR pair, and she can decode as there are at least $t+1$ correctly authenticated shares (given by the honest players). The honest dealer can then transmit her qubit.

Reconstruction of the secret is possible, when considering the initial encoding with a $t$ degree polynomial as an erasure code, and discarding shares which are not correctly authenticated 7 as these shares came from bad players. The reconstructor has at least $t+1$ points which define the secret (the points held by the honest players), so she can retrieve the original stat ${ }^{8}$. Secrecy follows from the no-cloning theorem and the ability of the reconstructor to reconstruct the right state. See [13] for a more complete proof of secure reconstruction.

\section{Lemma 4.2. If the dealer is faulty, the protocol is secure.}

\footnotetext{
${ }^{7}$ Formally, the first thing the receiver is doing is to open the authentication using the help of the classic TTP. The receiver then measures the last qubit (|ac $\rangle$ or $|r e\rangle)$.

${ }^{8}$ Actually the reconstructed state has exponentially good fidelity to the original state tensored with $|\mathrm{ac}\rangle$.
} 
Proof. Security in this context only means that after the sharing phase the state has been set, and can no longer be changed by the dealer. This means (for example) that the adversary knows the secret (he can choose it in the beginning of the protocol) and the only thing we should actually take care of is that the adversary will not be able to change the secret after the sharing phase (although he is allowed to prevent its decoding). To see that this is the case we follow the paths of the shares held by the players who are honest in the reconstruction phase. If the dealer passes the purity test done by the classical TTP, the two shared states have high fidelity to shared zeros. Therefore, in step 5 (the last step of the preparation), the honest players hold a state which has a high fidelity to an EPR-half encoded in a degree $t$ quantum polynomial code. Whether or not the dealer teleports a quantum state, there is an invariant: namely, that the states the honest players hold form $t+1$ points of some degree $t$ polynomial code, where each such point is authenticated by the dealer. (Note that if, for example, the dealer measures her half of the EPR pair, the state collapses, but we still have a polynomial code encoding a classical state where all the points are authenticated.) Therefore, in the reconstruction phase there are only two things which can happen:

1. The state reconstructed is the state encoded by the honest players' shares of the polynomial code, which was established in the sharing phase.

2. An authenticated share which does not sit on this polynomial code appears. In this case, w.h.p. no secret will be reconstructed (as there is no degree $t$ polynomial code which fits all the authenticated points) and the reconstructor knows that the dealer is faulty.

\section{Verifiable Quantum Secret Sharing}

Verifiable Quantum Secret Sharing is also a protocol with two phases. In the first phase, the dealer shares a quantum state (the secret) among all the players, such that the faulty players have no information about the state. In the second phase, the quantum data is sent to a reconstructor, who reconstructs the secret. We demand that the value which the reconstructor reconstructs is set during the sharing phase of the algorithm. As before, we only want our protocol to succeed with high probability.

The main difference between VQSS and WQSS is the dealer's capability to ruin the secret after it has been shared. Our main technique in solving this problem is based on the 2-Good-Trees of [8, 12] or the VQSS of [23]. The idea is to share a secret using a WQSS, and then share each one of the shares using WQSS. This means that the faulty players no longer have control of their shares. They can eliminate their shares by causing the WQSS reconstruction to fail, but cannot change them to some other state which could spoil the dealer's original state. Note that one of the last steps of the protocol we present, in which the authentication of the first layer is removed, is only needed for the full MPQC protocol.
We give a definition of Verifiable Quantum Secret Sharing using a TTP:

1. The dealer $D$ sends $T T P$ a state $\rho$, or no state at all. If D did not send a state the TTP notifies all the players that this is the case and the protocol ends.

2. Otherwise, at the reconstruction phase, a reconstructor $R$ is chosen and the TTP sends her the state.

Protocol As in WQSS, the sharing phase will have a long preparation part and then a simple sharing part. During the preparation the dealer will use a temporary authentication key $k_{\text {dealer }}$ in addition to the standard authentication channels we've established. As a preliminary step to the algorithm an authenticated channel is created with this key, and the key will be revealed to all the players at the end of the preparation. To sum up, the protocol for VQSS will demand two kinds of secret authentication keys:

1. The dealer has a temporary key $k_{\text {dealer }}$ for the first level of the tree. When sharing more than one secret in the MPQC each secret will have a new random key.

2. Each player $P_{i}$ will have a constant secret authentication key $k^{i}$ for the second level of the tree. These keys will be constant throughout MPQC.

In addition, each authentication has a random encryption key $x$ associated with it, as usual a different one for each state.

We give the detailed VQSS protocol in appendix C Briefly, it consists of the following steps:

1. The dealer shares encoded zero states using WQSS, and then each player further shares the state he receives, again using WQSS. The players and the classical TTP collectively check whether the states have been correctly shared and if they are in fact zeros.

2. The players use the shared zero states to create a shared EPR pair. Half of it is decoded and returned to the dealer.

3. Remove the top-level authentication (which uses the temporary key $k_{\text {dealer }}$ ) from the EPR pair using transversal Clifford group operations.

4. The dealer teleports his state through the EPR pair.

We note that it is possible to perform transversal Clifford operations between two shared secrets. The proof of this is very similar to the possibility of performing transversal Clifford operations on coded states. The only subtle point is that secrets shared by different dealers are actually protected in the same way (as we remove $k_{\text {dealer }}$ ).

We describe the reconstruction protocol here, and defer the security proof of both protocols to appendix $[$ Like the 
sharing protocol the reconstruction uses a one-time authentication key $k_{\text {reconstructor }}$ (with appropriate authenticated quantum channels), and the same player keys $k^{i}$.

Protocol Reconstruct-VQSS (Reconstructor R, Key for reconstruction $k_{\text {reconstructor }}$, Player Keys $k^{i}, 1 \leq i \leq n$ )

1. Reconstructor: Create an EPR pair and share it as in Share-VQSS. This includes a tree of height 2 and taking out the top level authentication, but excludes the final teleportation step.

2. All players and Reconstructor: Use quantum teleportation on the previously shared secret and on the reconstructor's shared EPR pair half to transfer the secret to the reconstructor's EPR-half which is still held by her. This is possible as after the removal of $k_{\text {reconstructor }}$ the codes of the dealer and reconstructor are actually identical, and it thus is possible to perform Clifford operations between their secrets.

Again we begin by assuming that the reconstructor is honest. We have two lemmas which together prove the security of the combined sharing-reconstruction protocol.

Lemma 5.1. If the dealer is honest, with exponentially good probability the faulty players cannot affect the reconstruction of the secret. Moreover, no player but the receiver learns anything (in the information theoretic sense) about the secret.

Lemma 5.2. If the dealer is faulty, with exponentially good probability he can not change the secret he shared. Moreover the faulty players do not learn $k^{i}$ values for honest players.

Both lemmas are proved in appendix $\mathrm{C}$

If the reconstructor is faulty, the only secret we are trying to protect is the player keys $k^{i}$. Their security stems from the security of performing Clifford group operations on coded states.

\section{Multiparty Quantum Computation}

Our VQSS scheme already resembles Multiparty Quantum Computation in the ability to share a few secrets in parallel (all with the same player authentication keys $k^{i}$ ), and use Clifford operations between them. In order to complete this to a Multiparty Quantum Computation we need to add a Toffoli gate. We base the creation of the Toffoli gate on a shared Toffoli state a la [24], and the sharing of our Toffoli state on the ideas of the Power-Tables in [23].

Let $\frac{1}{p} \sum_{a, b}|a, b, a b\rangle$ be the Toffoli State. It is well-known that it is possible to perform a Toffoli on any state using Clifford operations and this state.
Sharing the Toffoli state can be done by our protocol for VQSS. We begin by sharing many Toffoli states which are only polynomially good 9 and then use a fault-tolerant multiparty computation (for example [1]) to create an exponentially good Toffoli state from an encoded zero. The polynomially good states are created by some arbitrary player. If the player fails to create polynomially good Toffoli states she is faulty and is kicked out of the protocol.

Protocol Create-Toffolis (Arbitrary Dealer $P_{i}$, Player Keys $k^{i}$ for $1 \leq i \leq n$ )

1. $P_{i}$ : Share a polynomially large number of Toffoli states.

2. All players: Run state tomography on all but a polynomial fraction of the shared states to check the states sent by $P_{i}$. If $P_{i}$ is caught not sending Toffoli states, she is kicked out. Otherwise, the states sent have a polynomial fidelity to the Toffoli state.

3. All players: Using the states which were left (many states were opened up in the previous step), create an error correcting computation which creates a Toffoli state with exponential fidelity from the polynomially good Toffoli states. This can be done using the protocol described below, which uses standard techniques of noisy computation such as [1].

In appendix $\mathrm{D}$ we present a protocol especially designed to purify Toffoli states.

\section{Simulations of the protocol}

Proving the Universal Composability of our algorithm may seem at first like a daunting task. Surprisingly, this is not the case, and the simulation turns out to be almost trivial. We sketch the main details of the simulation in the full version. Here we simply note a few key features which we use:

1. We rely heavily on the universal composability of classical multiparty computation, and therefore use an ideal Classical Trusted Third Party (called here $C-T T P$ to stress that it's classical) in our simulation.

2. The preparation step of the protocol is generic and independent of the inputs (and even the function we evaluate). This makes its simulation trivial.

3. Using teleportation enables us to avoid passing quantum data in the input and output phases of the protocol. Instead, the players only pass (and obtain) classical data

\footnotetext{
${ }^{9}$ It might be possible to use Bravyi and Kitaev's technique in [5] and thus obtain exponential fidelity after passing a constant barrier. However, using their technique would require us to describe the entire calculation in $F_{2^{q}}$ instead of in $Z_{p}$ as we did here.
} 
to (and from) the $C-T T P$. Moreover, this data is uniformly distributed and independent of the quantum data in the computation.

4. For any group containing $\leq t$ players, the results of any transversal measurement done during the computation are random, uniformly distributed, and independent of the encoded quantum state which is being measured.

\section{Acknowledgments}

M.B. and A.H. were partially supported by an Israel Science Foundation research grant and by an Israel Ministry of Defense research grant. D.G. is supported by CIAR and NSERC of Canada.

\section{References}

[1] D. Aharonov and M. Ben-Or, "Fault tolerant quantum computation with constant error rate," quant-ph/9906129. Preliminary version in STOC '97. Submitted to SIAM J. Comp. .

ABDR04 A. Ambainis, H. Buhrman, Y. Dodis and H. Roehrig Amb04

[2] B. Awerbuch, B. Chor, S. Goldwasser and S. Micali, "Verifiable Secret Sharing and Achieving Simultaneity in the Presence of Faults,' In FOCS '85, 383-395, 1990.

ACGM85 B. Awerbuch, B. Chor, S. Goldwasser and S. Micali,

[3] Howard Barnum, Claude Crépeau, Daniel Gottesman, Adam Smith and Alain Tapp, "Authentication of Quantum Messages," In FOCS'02, 449-458, 2002.

[4] M. Ben-Or, S. Goldwasser and A. Wigderson, "Completeness Theorems for Non Cryptographic Fault-Tolerent Distributed Computation,” In STOC '88, pp. 1-10, 1988.

[5] S. Bravyi, and A. Kitaev , "Universal Quantum Computation Based on a Magic States Distillation," quant-ph/0403025 2004.

[6] M. Ben-Or, and D. Mayers , "General Security Definition and Composability for Quantum and Classical Protocols," quant-ph/0409062, 2004.

[7] Ran Canetti, "Universally Composable Security: A New Paradigm for Cryptographic Protocols,' In FOCS '01, pp. 136-145, 2001.

[8] D. Chaum, C. Crépeau and I. Damgård, "Multiparty Unconditionally Secure protocols," In STOC '88, pp. 11-19, 1988.

[9] A. M. Childs, "Secure assisted quantum computation," Quantum Information and Computation, 5: 456-466, 2005, quant-ph/0111046

[10] R. Cleve, "Limits on the Security of Coin Flips when Half the Processors Are Faulty,” In STOC '86, pp. 364-369.

[11] R. Cleve, D. Gottesman, and H.K. Lo, "How to share a quantum secret," Physical Review Letters, 83: 648-651, 1999.

[12] C. Crépeau, D. Gottesman and A. Smith, "Secure Multi-party Quantum Computing,” In STOC '02, pp. 643-652, 2002.
[13] C. Crépeau, D. Gottesman and A. Smith, "Approximate Quantum Error-Correcting Codes and Secret Sharing Schemes", In EUROCRYPT 2005, LNCS 3494, Jan 2005, Pages 285-301.

[14] D. Dolev, C. Dwork, O. Waarts and M. Yung, "Perfectly Secure Message Transmission,” In FOCS '90, 36-45, 1990.

[15] G. M. D'Ariano, D. Kretschmann, D. Schlingemann and R. F. Werner "Quantum Bit Commitment Revisited: the Possible and the Impossible," In quant-ph/0605224, 2006.

[16] O. Goldreich, S. Micali and A. Wigderson, "How to Play any Mental Game or A Completeness Theorem for Protocols with Honest Majority,' In STOC '87, 218-229, 1987.

[17] S. Goldwasser, "Multi-Party Computations: Past and Present," in PODC '97, 1-6, 1997.

[18] P. Hayden, D. Leung, D. Mayers, "Universal Composability for Quantum Authentication," unpublished.

[19] M. Nielsen and I. Chuang, Quantum Computation and Quantum Information, Cambridge University Press, 2000.

[20] E. Kushilevitz, Y. Lindell and T. Rabin. "InformationTheoretically Secure Protocols and Security Under Composition,” In STOC '06, 2006.

[21] R. Köenig and R. Renner , "A de Finetti Representation for Finite Symmetric Quantum States," In Journal of Mathematical Physics 46, 122108, 2005, quant-ph/0410229

[22] T. Rabin, "Robust Sharing of Secrets when the Dealer is Honest or Cheating," J. ACM, 41(6), 1089-1109, November 1994.

[23] T. Rabin and M. Ben-Or, "Verifiable Secret Sharing and Multiparty Protocols with Honest Majority (Extended Abstract)," In STOC '89, 73-85, 1989.

[24] P. W. Shor, "Fault-tolerant quantum computation," In FOCS '96, 56-65, 1996, quant-ph/9605011

\section{A A Zero Purity Test}

We describe a simple zero purity test for states $\left|\phi_{0}\right\rangle, \ldots\left|\phi_{w}\right\rangle$ (for some $w$ ), to test if they are all authenticated correctly and are encoding the qudit $|0\rangle$. Choose $a_{0}, \ldots a_{w} \in_{R}$ $\{0,1, \ldots, p\}$ and calculate the transformation $\sum a_{i}\left|\phi_{i}\right\rangle \rightarrow$ $\left|\phi_{0}\right\rangle$ where the sum is done by using SUM. Then, open up (i.e., measure) the new state $\left|\phi_{0}\right\rangle$. Correct the state you have using the keys, applying shifts and multiplications. You should be holding a polynomial of degree $\leq d$ with free coefficient 0 . Run the same check (with new random numbers and without $\left|\phi_{0}\right\rangle$ which was already spent) on the Fourier transform of the states, although now the free coefficient is random. Iterating this $s$ times spends $2 s$ states and gives the desired fidelity. All the operations (multiplying with constants, SUM's and measurements) are done with the help of the classical $T T P$, as only the $T T P$ holds the classical keys.

In a way similar to the definitions of authenticated states, let $M$ be the Hilbert space which holds the remaining states $\left|\phi_{0}\right\rangle, \ldots,\left|\phi_{w-2 s}\right\rangle$, let $|\psi\rangle=\mid$ Auth $\left.(0)\right\rangle \otimes|A u t h(0)\rangle \otimes \cdots \otimes$ $|A u t h(0)\rangle \in M$, and let $\mathrm{V}$ be a Hilbert space of dimension 2 , with basis states $|\mathrm{ac}\rangle,|\mathrm{re}\rangle$. Define projectors

$$
P_{1}^{|\psi\rangle}=|\psi\rangle\left\langle\psi\left|\otimes I_{V}+I_{M} \otimes\right| \mathrm{re}\right\rangle\langle\mathrm{re}|-| \psi\rangle\langle\psi|\otimes| \mathrm{re}\rangle\langle\mathrm{re}|
$$




$$
P_{0}^{|\psi\rangle}=\left(I_{M}-|\psi\rangle\langle\psi|\right) \otimes|\mathrm{ac}\rangle\langle\mathrm{ac}|
$$

Lemma A.1. The result of the zero purity test has fidelity $1-$ $O\left(p^{-s}\right)$ to the space spanned by $P_{1}^{|\psi\rangle}$. If the adversary did not change the authenticated state the output will be $|\psi\rangle \otimes$ $|\mathrm{ac}\rangle$.

The proof of the lemma is deferred to the final version.

\section{B Weak Quantum Secret Sharing}

In this appendix, we give the detailed protocol for sharing states using WQSS.

Protocol Weak-Quantum-Secret-Sharing (Dealer D, Dealer Key $k_{\text {dealer }}$

1. Preparation Dealer $D$ : Encode many $(2 s+2$, where $\mathrm{s}$ is the security parameter) zeros using the quantum polynomial code of degree $t$ and length $n$. For each zero encoded send the $i$ th share to $P_{i}$, using the authenticated channel established in section 3 Note that the authenticated channel is used with the same $k_{\text {dealer }}$ all the time, but with different $x$ 's.

2. All players and classical-TTP: Using random numbers generated by the classical $T T P$, the players perform transversal random sums, both in the standard and the Fourier basis. The players measure $2 s$ of their shares ( $s$ checks in each basis) and send the results to the classical TTP.

3. Classical-TT P: Discard values which do not authenticate correctly. These values must come from bad players (we are using verified authenticated channels).

4. Classical-TTP: If errors are detected in the outer polynomial code the dealer is faulty. All players are informed and the protocol is aborted.

5. All players: The players collectively generate an EPR pair from the two remaining zeros and send one half of the pair to the dealer.

6. Sharing Dealer: Decode the EPR-half you have 11 and using quantum teleportation send the secret, giving your measurement results to the TTP. This results only in a change of keys - the players do not need to act on their states or manipulate any new information.

\footnotetext{
${ }^{10}$ Underlined and words are the name of the phase. The phase continues until a new phase begins.

${ }^{11}$ Note that the dealer can perform this step only because he knows all the keys he used in step 1 .
}

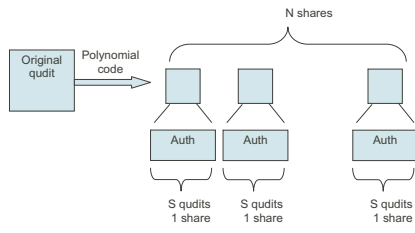

Figure 1: Schematic of Weak QSS

\section{Details of VQSS}

Below is the detailed protocol for sharing in VQSS:

Protocol Share-VQSS (Dealer $D$, Secret $\psi$, Key for the secret $k_{\text {dealer }}$, Player Keys $k^{i}$ for $1 \leq i \leq n$ )

1. Preparation Phase Dealer: Prepare many $(2 s+2)$ zeros and encode them with the polynomial code of degree $t$ and length $n$. Send the $i$ 'th share $\left(R_{i}\right)$ to the $i$ 'th player using the authenticated channel using key $k_{\text {dealer }}$.

2. Player $P_{i}$ : Take each state shared by the dealer, and share it using WQSS and your key $k^{i}$. Mark the $j$ 'th share as $R_{i, j}$. Note that for each zero-share $P_{i}$ got from the dealer, she has to generate $2 s+2$ new zeros.

3. All players and classical-TTP: Perform transversal random sums in both standard and Fourier basis to check that the zeros shared by the dealer are OK. All results are sent to the classical TTP. Note that the shares $R_{i, j}$ are being manipulated here, and that $2 s$ of the original zeros are being spent.

4. Classical-TTP, operating on the measurement results that were given by the players: If a measurement result on $R_{i, j}$ is not authenticated, $P_{j}$ is faulty and this result should be ignored. If $R_{i}$ is not properly reconstructed as a state authenticated by the dealer, $P_{i}$ is faulty; inform all players of this. If the top level does not decode to $|0\rangle$ (when ignoring faulty $R_{i}$ 's), the dealer is faulty. If the dealer is faulty the classical TTP tells that to all the players and the dealer is kicked out.

5. Generating EPR All players $P_{j}$ with the help of classical-TTP: Using transversal operations on two shared zeros generate an EPR pair. This is done by acting on $R_{i, j}$ shares.

6. Sending the EPR Half to the Dealer Player $P_{j}$ : Send half of the pair created from the $R_{i, j}$ 's to $P_{i}$.

7. Player $P_{i}$ : Using the shares you received (using only ones which are correctly authenticated by you) decode 


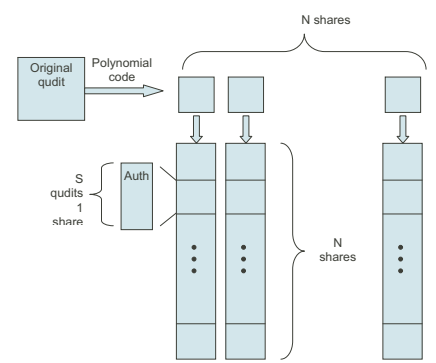

Figure 2: Schematic of VQSS

$R_{i}$ and send it back to the dealer. Note that $P_{i}$ knows $k^{i}$, and does not need the help of the classical TTP here.

8. Dealer: Decode the state you received, discarding any incorrectly authenticated shares.

9. Getting Rid of $k_{\text {dealer }}$ Players $P_{j}$ with the help of classical-TTP: Using transversal Clifford operations remove the top level authentication. This is possible as each $R_{i}$ was authenticated and the qudits were shared by $P_{i}$. Using transversal operation on these qudits we can decode $R_{i}$ and leave this coordinate protected only by WQSS using $k^{i}$. This step is not essential for VQSS but only for MPQC.

10. Sharing Dealer: Share your secret using quantum teleportation, passing measurement results to the classical $T T P$. This only results in changes of $x$ keys (just like the sharing in WQSS).

\section{Purifying Toffoli States}

We present an algorithm to purify Toffoli states, which is interesting in its own right. Each Toffoli state allows us to perform one Toffoli gate; a perfect Toffoli state gives a perfect Toffoli gate, whereas a state with error $\epsilon$ produces a gate which is $O(\epsilon)$ away from a correct Toffoli gate. Then we can use techniques of fault-tolerant quantum computation to turn these noisy gates into an exponentially more reliable one.

Choose some $m$ and $d$, with $m=3 d+1, p>m, m=$ $O(s)$.

Let $H_{\text {good }}$ be the space spanned by $m$ Toffoli states which were affected by at most $m / 8$ non-identity Pauli operations. Then any $m$ Toffoli states coming from players who have passed the polynomial state tomography phase will have fidelity $1-2^{-O(s)}$ to their projection on $H_{\text {good }}$. We now show how to distill a single exponentially good Toffoli state from the $m$ states.
Let $\beta_{1}, \ldots \beta_{m} \in \mathbb{Z}_{p}$ be distinct nonzero points. Look at the state

$$
\begin{aligned}
\sum_{a, b} \sum_{\substack{d e g(f) \leq d \\
f(0)=a}} \sum_{\substack{d e g(g) \leq d \\
g(0)=b}} \sum_{\substack{d e g(h) \leq 2 d \\
h(0)=0}}\left(\left|f\left(\beta_{1}\right), \ldots, f\left(\beta_{m}\right)\right\rangle\right. \\
\left.\otimes\left|g\left(\beta_{1}\right), \ldots, g\left(\beta_{m}\right), h\left(\beta_{1}\right), \ldots, h\left(\beta_{m}\right)\right\rangle\right) .
\end{aligned}
$$

Note that this state can be created exactly by Clifford operations (which are free in the model we discuss). Now, for $1 \leq i \leq m$ use a polynomially good Toffoli state (one of the $m$ states we have left after the first part of the purification) to perform a Toffoli gate on coordinates $i, m+i, 2 m+i$. If the Toffoli states were ideal this should result in the state $|\tau\rangle=$

$$
\begin{array}{r}
\sum_{a, b} \sum_{\begin{array}{c}
d e g(f) \leq d \\
f(0)=a
\end{array}} \sum_{\substack{d e g(g) \leq d \\
g(0)=b}} \sum_{\substack{d e g(h) \leq 2 d \\
h(0)=a b}}\left(\left|f\left(\beta_{1}\right), \ldots, f\left(\beta_{m}\right)\right\rangle\right. \\
\left.\otimes\left|g\left(\beta_{1}\right), \ldots, g\left(\beta_{m}\right), h\left(\beta_{1}\right), \ldots, h\left(\beta_{m}\right)\right\rangle\right) .
\end{array}
$$

As the entire $m$ states have exponentially good probability to a state in $H_{\text {good }}$, with exponentially good probability we can correct $d / 2$ mistakes on each one of the three codes, and be in a state which has exponential good fidelity to $|\tau\rangle$. Decoding the state using only Clifford group operations we get a state with exponential fidelity to $\frac{1}{p} \sum_{a, b}|a, b, a b\rangle$, which is the Toffoli State.

Note that the error rate in this purification step drops from $\eta$ down to $\eta^{O(d)}$. We can select a (polynomially) large $d$ or iterate the procedure with smaller $d$ to obtain the desired fidelity. 